\title{
Dynamic modelling of die melt temperature profile in polymer extrusion
}

\section{Document Version}

Accepted author manuscript

Link to publication record in Manchester Research Explorer

\section{Citation for published version (APA):}

Abeykoon, C., Kelly, A. L., Martin, P. J., \& Li, K. (2013). Dynamic modelling of die melt temperature profile in polymer extrusion. In host publication (pp. 2550-2555)

\section{Published in:}

host publication

\section{Citing this paper}

Please note that where the full-text provided on Manchester Research Explorer is the Author Accepted Manuscript or Proof version this may differ from the final Published version. If citing, it is advised that you check and use the publisher's definitive version.

\section{General rights}

Copyright and moral rights for the publications made accessible in the Research Explorer are retained by the authors and/or other copyright owners and it is a condition of accessing publications that users recognise and abide by the legal requirements associated with these rights.

\section{Takedown policy}

If you believe that this document breaches copyright please refer to the University of Manchester's Takedown Procedures [http://man.ac.uk/04Y6Bo] or contact uml.scholarlycommunications@manchester.ac.uk providing relevant details, so we can investigate your claim.

\section{OPEN ACCESS}




\title{
Dynamic Modelling of Die Melt Temperature Profile in Polymer Extrusion
}

\author{
Chamil Abeykoon, Adrian L. Kelly, Peter J. Martin, Kang Li
}

\begin{abstract}
The extrusion process is one of the main methods of processing polymeric materials and thermal homogeneity of the process output presents a major challenge for high quality extruded products. Therefore, accurate process thermal monitoring and control are highly desirable. However, most of the industrial extruders use conventional single point thermocouples for thermal monitoring although their measurements are highly affected by barrel metal wall temperature. Moreover, it has been shown that the melt temperature changes considerably with the die radial position and point based measurements are not sufficient to determine the actual process thermal stability and hence to control the thermal homogeneity of melt output. Conversely, the majority of process thermal control methods are based on linear models and are not capable of dealing with process nonlinearities. In this work, a die melt temperature profile was monitored by a thermocouple mesh technique and the data obtained was used to formulate a new nonlinear dynamic model to predict the die melt temperature profile in a single screw extruder. The model is in good agreement with the measured data and offers a promising thermal monitoring technique which can be used in real-time for a thermal profile based control framework in polymer extrusion.
\end{abstract}

Index Terms - Polymer extrusion, Process monitoring, Thermal homogeneity, Melt temperature profile, System identification, Dynamic modelling.

\section{INTRODUCTION}

Despite significant developments in polymer extrusion over the last few decades, process thermal monitoring and control still remains an issue. Process operators have to face challenges in achieving the required thermal quality of the melt output with prevailing knowledge and technology. Achieving good thermal stability is a major requirement to form high quality extruded products. Even small variations in melt temperature can cause poor product quality [1]. Therefore, continuous monitoring of process thermal stability is invaluable for advanced process control to form a high quality melt output. More details on the process operation and mechanisms of polymer extrusion can be found in the literature [2]. As revealed by the previous studies [3][9], the thermal homogeneity of the melt is considerably affected by the process settings and melt flow temperature is different at different radial locations of the die. Therefore, the study of the entire melt temperature profile as a measure of the process thermal stability is more appropriate than a single point or a bulk measurement to ensure high quality

Chamil Abeykoon and Adrian L. Kelly is with the School of Engineering, Design and Technology, University of Bradford, Bradford, BD7 1DP, UK, E-mail: Y.Abeykoon@bradford.ac.uk, yabeykoon01@qub.ac.uk

P. J. Martin is with the the School of Mechanical and Aerospace Engineering, Queen's University Belfast, Belfast, BT9 5AH, UK

$\mathrm{K} . \mathrm{Li}$ is with the School of Electronics, Electrical Engineering and Computer Science, Queen's University Belfast, Belfast, BT9 5AH, UK extruded products. Unfortunately, it is quite difficult to monitor a die melt temperature profile within a production environment and most of the extruders are instrumented only with conventional wall mounted thermocouples for thermal measurements. These are highly affected by the barrel wall temperature and they are not capable of measuring a melt temperature profile or detecting rapid variations in melt temperature [10], [11]. As alternatives to the point/bulk measurements, some of the thermal profile measurement methods (e.g. a thermocouple mesh [8], a fluorescence technique [12]) were proposed, but these are not yet robust enough to use in a production environment due to the constraints such as their complexity, limited durability, access requirements, disruptive effects on melt flow and output, etc. However, some of these techniques have been used to gatherer valuable process thermal information in a research setting.

\section{A. Melt temperature modelling}

As reported in the literature, little work has been carried out so far on the modelling of an extrusion melt temperature profile under static or dynamic conditions. Instead, reports can be found on the development of static or dynamic models [13]-[20] to predict a single point or bulk melt temperature. Essentially, some of these modelling attempts are based on first principles while others are data driven models based on point or bulk melt temperature measurements taken from thermocouples fixed at the end of the extruder barrel or the die. Accuracy of the first principle models is constrained due to numerous simplifying assumptions and also they are computationally too expensive to implement for real-time which limits their practical applications [21]. While some of the other point/bulk thermal models may be used in practice, these provide no detailed information on the actual process thermal stability. Furthermore, most of these existing thermal models are linear models and hence these may not be sufficient to represent the nonlinear processing behaviour.

A previous study by the authors [5], [22] developed a static model to predict the die melt temperature profile in single screw extrusion. Melt temperatures at different radial locations of the die were predicted from readily measurable process variables (i.e. screw speed and barrel set temperatures) and good agreement was achieved between the experimental and model predicted die melt temperature profiles. To our knowledge, no any other melt temperature profile modelling work was found in the literature.

In this work, a thermocouple mesh [8] was used to measure temperature profiles across the die melt flow over different processing conditions. The data obtained was used 
to develop a nonlinear dynamic model to predict the die melt temperature profile. The study was only focused on the melt temperature of single screw extruders with a single material.

\section{EQUIPMENT \& PROCEDURE}

All measurements were carried out on a $63.5 \mathrm{~mm}$ diameter (D) single screw extruder (Davis Standard BC-60). A barrier flighted screw with a spiral Maddock mixer and a 2.5:1 compression ratio (Feed or Solids conveying: $5 \times \mathrm{D}$, Melting or Compression: $13 \times \mathrm{D}$, Metering or Pumping: $6 \times \mathrm{D}$ ) was used to process the material. The extruder was fitted with an adaptor by using a clamp ring prior to a short capillary die with a $6 \mathrm{~mm}$ bore as shown in Figure 1.

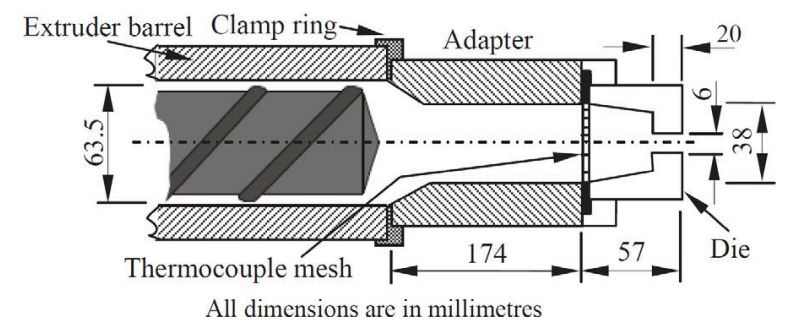

Fig. 1. Extruder die, adapter and thermocouple mesh

The extruder barrel has four separate temperature zones and another three separate temperature zones are also available at the clamp ring, adapter and die. Each of these temperature zones is equipped with a separate temperature controller which allows individual control of the set temperature. The extruder barrel dimensions and arrangement of heaters are shown in Figure 2.

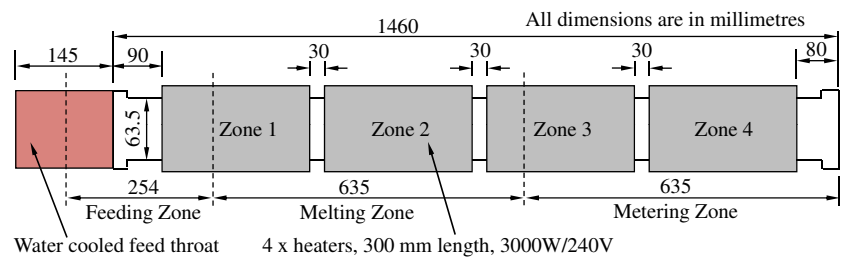

Fig. 2. BC-60 extruder barrel arrangement and dimensions

Melt temperatures of the different radial locations of the melt flow at the end of the adapter (denoted as die melt temperatures throughout this paper) were measured using a thermocouple mesh placed in-between the adapter and the die as shown in Figure 1. As it was previously confirmed by Kelly et al. [7], [8], the die melt temperature measurements are symmetrical across the thermocouple mesh centreline. Therefore, seven thermocouple junctions (i.e. with seven positive and one negative thermocouple wires) were placed asymmetrically across the melt flow along the diameter of the mesh as shown in Figure 3, and this asymmetric placement of wires gave the opportunity to increase the number of effective temperature measurements across the melt flow by mirroring them over the centreline to obtain the complete die melt temperature profile. The die wall set temperature was used as the melt temperatures at the $\pm 19 \mathrm{~mm}$ radial positions. Then, the final temperature profile was obtained by 15 radial positions (distance from the melt flow centreline to each radial position: $0 \mathrm{~mm}, \pm 3 \mathrm{~mm}, \pm 4.5 \mathrm{~mm}, \pm 8.8 \mathrm{~mm}$, $\pm 11 \mathrm{~mm}, \pm 14.7 \mathrm{~mm}, \pm 16.5 \mathrm{~mm}$ and $\pm 19 \mathrm{~mm}$ ) across the melt flow as illustrated in Figure 3.

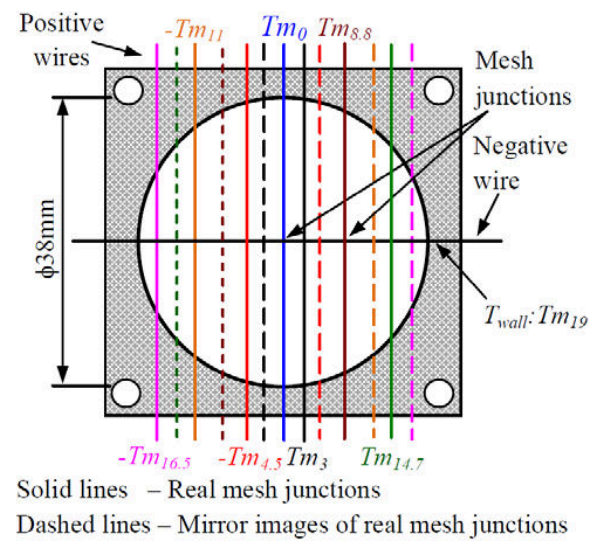

Fig. 3. The thermocouple mesh arrangement

A data acquisition programme developed in LabVIEW was used to communicate between the experimental instruments and a PC. Screw speed and all temperature signals were acquired at $10 \mathrm{~Hz}$ using a 16-bit DAQ card (National Instruments (NI) PCI-6035E) through a thermocouple connector box (NI TC-2095) and a low-noise signal conditioning box (NI SCXI-1000). Experimental trials were carried out on a virgin high density polyethylene (HDPE), (ExxonMobil HYA 800), (density: $0.961 \mathrm{~g} / \mathrm{cm}^{3}$, melt flow index (MFI): $0.7 \mathrm{~g} / 10$ min @ $\left.\left(190{ }^{\circ} \mathrm{C}, 2.16 \mathrm{~kg}\right)\right)$. The extruder barrel temperature settings were fixed as described in Table I under three different set conditions denoted as A (high temperature), B (medium temperature) and $\mathrm{C}$ (low temperature).

TABLE I

EXTRUDER BARREL TEMPERATURE SETTINGS

\begin{tabular}{|c|c|c|c|c|c|c|c|}
\hline \multirow{3}{*}{$\begin{array}{c}\text { Temperature } \\
\text { settings }\end{array}$} & \multicolumn{7}{|c|}{ Set temperatures $\left({ }^{\circ} \mathrm{C}\right)$} \\
\hline & \multicolumn{4}{|c|}{ Barrel Zones } & \multirow{2}{*}{ Clamp Ring } & \multirow{2}{*}{ Adapter } & \multirow{2}{*}{ Die } \\
\hline & 1 & 2 & 3 & 4 & & & \\
\hline A & 110 & 130 & 180 & 230 & 230 & 230 & 230 \\
\hline B & 105 & 125 & 175 & 215 & 215 & 215 & 215 \\
\hline $\mathrm{C}$ & 100 & 120 & 170 & 200 & 200 & 200 & 200 \\
\hline
\end{tabular}

The experiments were started with the temperature setting A and data was recorded with the screw stationary for 1 minute. Then, the screw speed was increased up to $90 \mathrm{rpm}$ with random steps of between $\pm 5-40 \mathrm{rpm}$ and in different barrel set temperatures with the extruder running for about 193 minutes continuously as shown in Figure 6. The extruder was allowed to stabilise for 15 minutes after each set temperature change while it was hold for about 7 minutes at each other different condition. All of these settings were selected in order to generate realistic processing conditions whilst covering the full operating range of the extruder (i.e. 0$100 \mathrm{rpm})$. This therefore allowed investigation of melting performance at low throughputs where melting is dominated by conduction from the barrel and screw, and intermediate and high throughputs where melting is primarily achieved by viscous shearing. Separate tests were carried out to collect the data for model training and validation. 


\section{EFFECTS OF PROCESS SETTINGS ON DIE MELT TEMPERATURE PROFILE}

As stated in section II, melt temperature profiles across the melt flow were observed and profiles at different barrel temperature settings are shown in Figure 4. The average values of the data collected for the last two minutes of each screw speed was used to plot these profiles.

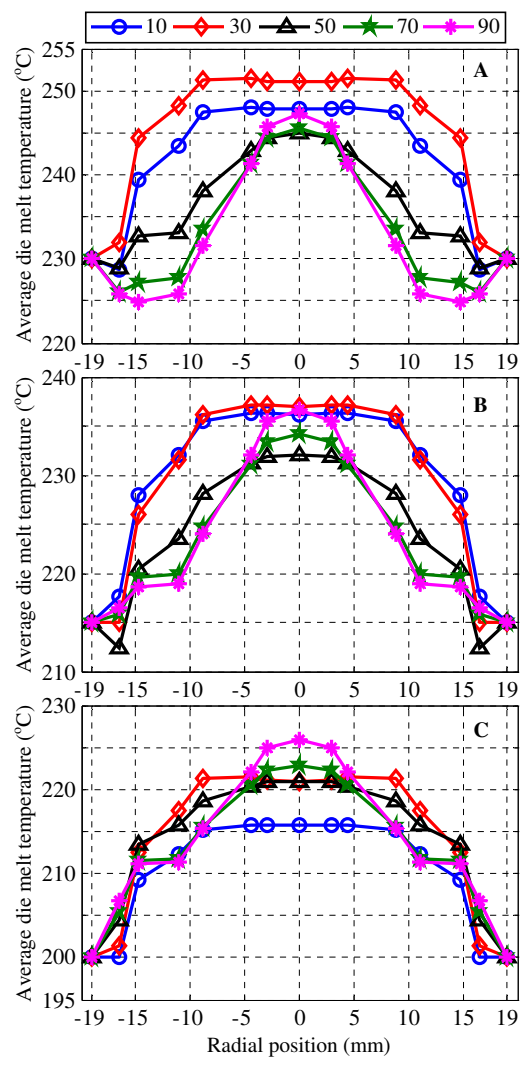

Fig. 4. Average die melt temperature profiles over the last 2 minutes at different processing conditions for tests A, B and C from 10-90 rpm

Ideally, these should be flat profiles under all processing conditions for better thermal homogeneity. It is clear that screw speed has a significant impact on the shape of the profiles and these effects differ with the barrel set temperatures as well. For example, the highest temperature level at test condition A has occurred at $30 \mathrm{rpm}$, but at test condition $\mathrm{C}$ both 70 and $90 \mathrm{rpm}$ temperatures are higher than the temperature at $30 \mathrm{rpm}$. In general, all temperature profiles have shifted down from test condition $A$ to $C$ which highlights another effect of barrel set temperatures on the level of the die melt temperature.

\section{MODELLING}

In general, the melt temperature $\left(T_{m, j}\right)$ at a particular die radial position $\left(R_{p, j}\right)$ which is $j \mathrm{~mm}$ away from the melt flow centre can be represented as a function of $\omega_{s c}, R_{p, j}$ and $T_{b}$ :

$$
T_{m, j}=f\left(\omega_{s c}, R_{p, j}, T_{b}\right)
$$

where $\omega_{s c}$ is the screw speed and $T_{b}$ represents the barrel set temperatures (subscript $b$ represents different barrel zones $\left.T_{1}-T_{4}\right)$. Six model inputs $\left(\omega_{s c}, R_{p, j}, T_{1}, T_{2}, T_{3}, T_{4}\right)$ and one output $\left(T_{m, j}\right),-19 m m \leq j \leq 19 \mathrm{~mm}$, were considered as illustrated in Figure 5. The set temperatures of the clamp

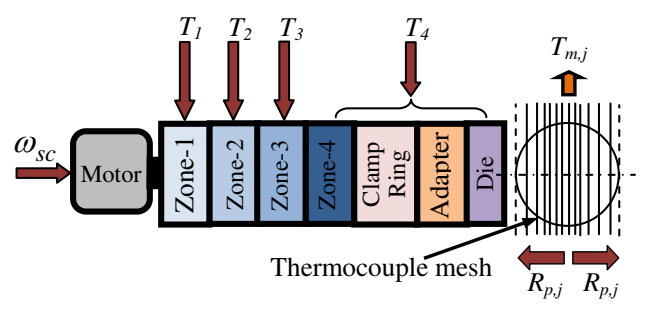

Fig. 5. Extruder model with selected inputs and output

ring, the adapter, and the die are always equal to $T_{4}$ in this study. If these values are different from $T_{4}$, it is possible to add them as three different model inputs.

\section{A. Model development and operation}

As a large amount of data was available, both training and validation data were down sampled to $1 \mathrm{~Hz}$ for the ease of data processing. The model predicts the melt temperature values of each radial position assigned by the radial position input. As shown in Figure 3, fifteen radial positions make a complete melt temperature profile across the die melt flow. The model estimates the melt temperature values of these fifteen points individually by only changing the radial position input while screw speed and barrel set temperatures remain constant. There are 25 different processing situations and each input signal contains 173,700 data points. The model input matrices for both training and validation data are shown in Figure 6.

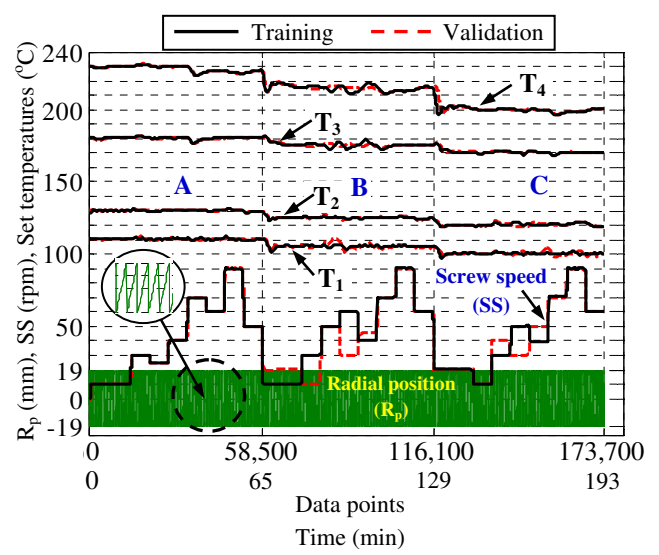

Fig. 6. Model input matrices

The model output contains the measured melt temperature at each radial position corresponding to the relevant inputs and the signal length is the same as the input signal length.

A nonlinear polynomial model was adopted to approximate the original system due to the linear models were not shown satisfactory performance. A two-stage algorithm [23], [24] was employed in the selection and refinement of the Linear-In-The-Parameters (LITP) models for this study. In the first stage, a fast recursive algorithm (FRA) was used for the selection of the model structure and for estimation of the model parameters. This solves the problem recursively and does not require matrix decomposition as is the case for 
orthogonal least squares (OLS) techniques [25]. Also, this FRA has proved that it has a lower computational complexity and a better numerical stability over OLS. However, the models developed include a constraint that the terms added later are based on previously selected ones. As a result, some of them may not have a significant contribution. Then, in the second stage a backward model refinement procedure was carried out to eliminate non-significant terms to build up a compact model. The significance of each selected model term was reviewed and compared with those remaining in the candidate term pool and all insignificant terms were replaced, leading to improved performance without increasing the model size.

\section{B. Fast recursive algorithm (FRA)}

Suppose a general nonlinear discrete-time dynamic multiinput-single-output (MISO) system can be expressed as:

$$
\begin{aligned}
y(t)= & f\left(y(t-1), y(t-2), \ldots y\left(t-n_{1}\right), \ldots y\left(t-n_{a}\right), u_{i}\left(t-n_{i k}\right),\right. \\
& \left.u_{i}\left(t-n_{i k}-1\right), \ldots u_{i}\left(t-n_{i k}-n_{1}\right), \ldots u_{i}\left(t-n_{i k}-n_{i b}\right)\right)
\end{aligned}
$$

where $y(t)$ is the system output at time $t, u_{i}(t), i=1, \cdots, m$ are the system inputs at time $t$ ( $m$ is the total number of inputs to the system), $n_{a}$ is the number of poles, $n_{i b}$ is the number of zeros plus 1 and $n_{i k}$ is the corresponding delays (i.e. number of input samples that occur before each input affects the output) of each input. By using a polynomial function, this equation can be approximated using a LITP model:

$$
y(t)=\sum_{i=1}^{M} \theta_{i} \varphi_{i}(\mathbf{x}(\mathbf{t}))+e(t)
$$

where $\varphi_{i}(\cdot), i=1, \cdots, M$ are all candidate model terms, $\mathbf{x}(\mathbf{t})=\left[u_{1}(t), \cdots, u_{m}(t)\right]^{T}$ is the model input vector, and $\mathrm{e}(\mathrm{t})$ is the modelling residue.

Suppose $\mathrm{N}$ data samples are used for model training, then (3) can be re-written as:

$$
\mathbf{y}=\boldsymbol{\Phi} \boldsymbol{\theta}+\mathbf{e}
$$

where $\mathbf{\Phi}=\left[\phi_{1}, \cdots, \phi_{M}\right] \in \Re^{N \times M}$ is the regression matrix with column vectors $\phi_{\boldsymbol{i}}=\left[\varphi_{i}(\mathbf{x}(1)), \cdots, \varphi_{i}(\mathbf{x}(N))\right]^{T}$, $\mathbf{y}=[y(1), \cdots, y(N)]^{T} \in \Re^{N}$ is the desired output, $\boldsymbol{\theta}=$ $\left[\theta_{1}, \ldots, \theta_{M}\right]^{T} \in \Re^{M}$ is the model coefficients and $\mathbf{e}=$ $[e(1), \cdots, e(N)]^{T} \in \Re^{N}$ is the residual vector.

The well known least-square method solves the problem by minimising the cost function:

$$
J(\boldsymbol{\theta})=\mathbf{e}^{T} \mathbf{e}
$$

and the corresponding solution is given by:

$$
\hat{\boldsymbol{\theta}}=\left(\boldsymbol{\Phi}^{\mathrm{T}} \boldsymbol{\Phi}\right)^{-1} \boldsymbol{\Phi}^{\mathrm{T}} \mathbf{y}
$$

However, due to the noise and correlations between a large number of regressors, the information matrix $\boldsymbol{\Phi}^{\mathrm{T}} \boldsymbol{\Phi}$ is always ill-conditioned in practice, which may lead to inaccurate calculation of the model coefficients $\hat{\boldsymbol{\theta}}$. Ridge regression can prevent this problem, but gives a biased solution. Therefore, use of a subset selection algorithm eliminates this problem by selecting the most relevant and significant terms.

The FRA utilised in this study can be presented by defining a recursive matrix, $\mathbf{M}_{k}$, and a residual matrix, $\mathbf{R}_{k}$ :

$$
\begin{aligned}
& \mathbf{M}_{\mathbf{k}} \triangleq \boldsymbol{\Phi}_{\mathbf{k}}^{\mathrm{T}} \boldsymbol{\Phi}_{\mathbf{k}} \quad k=1, \cdots, M \\
& \mathbf{R}_{\mathbf{k}} \triangleq I-\boldsymbol{\Phi}_{\mathbf{k}} \mathbf{M}_{\mathbf{k}}^{-\mathbf{1}} \boldsymbol{\Phi}_{\mathbf{k}}^{\mathrm{T}} \quad \mathbf{R}_{0} \triangleq I
\end{aligned}
$$

where $\boldsymbol{\Phi}_{k} \in \Re^{N \times k}$ contains the first $k$ columns of the full regression matrix $\Phi$, and the cost function in (5) can be rewritten as: $\quad J\left(\mathbf{P}_{k}\right)=\mathbf{y}^{\mathrm{T}} \mathbf{R}_{k} \mathbf{y}$

where $\mathbf{P}_{k}=\left[\mathbf{p}_{1}, \ldots, \mathbf{p}_{k}\right], k=1, \ldots, n, n$ represents the total number of terms included in the final model, and $\mathbf{p}_{i}$ represents the selected model terms from all the candidates of those remaining in the pool denoted as, $\phi_{i}, i=1, \cdots, M$.

In this forward stepwise selection, polynomial terms are selected one by one based on their contributions to the final model. As shown in [23], [24], if one more regressor $\phi_{j}$ from the candidate term pool is to be selected, the net contribution of $\phi_{j}$ to the cost function can be calculated as:

$$
\Delta J_{k+1}\left(\phi_{j}\right)=\mathbf{y}^{\mathrm{T}}\left(\mathbf{R}_{k+1}-\mathbf{R}_{k}\right) \mathbf{y}=\frac{\left(\mathbf{y}^{\mathrm{T}} \boldsymbol{\phi}_{j}^{(k)}\right)^{2}}{\boldsymbol{\phi}_{j}^{\mathrm{T}} \boldsymbol{\phi}_{j}^{(k)}}
$$

where $\phi_{j}^{(k)} \triangleq \mathbf{R}_{k} \phi_{j}, k+1 \leqslant j \leqslant M$. The above net contribution can be further simplified by defining an auxiliary matrix $\mathbf{A} \in \Re^{k \times M}$ and a vector $\mathbf{b} \in \Re^{M \times 1}$ with their elements given by:

$$
\begin{gathered}
a_{i, j} \triangleq \begin{cases}\left(\mathbf{p}_{i}^{(i-1)}\right)^{\mathrm{T}} \mathbf{p}_{j}, & 1 \leq j \leq k \\
\left(\mathbf{p}_{i}^{(i-1)}\right)^{\mathrm{T}} \boldsymbol{\phi}_{j}, & k<j \leq M\end{cases} \\
b_{i} \triangleq \begin{cases}\left(\mathbf{p}_{i}^{(i-1)}\right)^{\mathrm{T}} \mathbf{y}, & 1 \leq i \leq k \\
\left(\boldsymbol{\phi}_{i}^{(k)}\right)^{\mathrm{T}} \mathbf{y}, & k<i \leq M\end{cases}
\end{gathered}
$$

According to the properties of $\mathbf{R}_{k}$ ([23], [24]), $a_{k, j}$ and $b_{k}$ can be updated recursively:

$$
\begin{gathered}
a_{k, j}=\mathbf{p}_{k}^{\mathrm{T}} \boldsymbol{\phi}_{j}-\sum_{l=1}^{k-1} a_{l, k} a_{l, j} / a_{l, l} \\
k=1, \cdots, n, \quad j=1, \cdots, M . \\
b_{k}=\mathbf{p}_{k}^{\mathrm{T}} \mathbf{y}-\sum_{l=1}^{k-1} a_{l, k} b_{l} / a_{l, l} \quad k=1, \cdots, n .
\end{gathered}
$$

By substituting (13) and (14) into (10), the net contribution of $\phi_{j}, j=k+1, \cdots, M$ to the cost function can be given as:

$$
\Delta J_{k+1}\left(\phi_{j}\right)=-\frac{b_{j}^{2}}{a_{j, j}}
$$

The model term that gives the largest contribution is then selected, and this procedure is continued until some criterion is met (e.g., Akaike's information criterion (AIC) [26]) or a pre-set maximum number of terms are selected. To further reduce the calculation complexity in term selection, at the $(k+1)$-th step, $a_{j, j}^{(k+1)}$ and $b_{j}^{(k+1)}(j=k+1, \cdots, M)$ can be updated recursively instead of being computed from (13) and (14):

$$
\begin{aligned}
& a_{j, j}^{(k+1)}=a_{j, j}^{(k)}-a_{k, j}^{2} / a_{k, k} \\
& b_{j}^{(k+1)}=b_{j}^{(k)}-a_{k, j} b_{k} / a_{k, k}
\end{aligned}
$$

At the end of each selection, these terms are updated and stored for the next comparison/selection. After a satisfactory 
model has been constructed, the model coefficients can be computed recursively.

$$
\hat{\theta}_{j}=\left(b_{j}-\sum_{i=j+1}^{k} \hat{\theta}_{i} a_{j, i}\right) / a_{j, j}, \quad j=k, k-1, \cdots, 1 .
$$

where the terms $a_{j, j}$ and $b_{j}$ in (18) are similar notations for the $a_{j, j}^{(k)}$ and $b_{j}^{(k)}$ terms used above. Finally, a more compact model (i.e. terms and coefficients) which includes only the most significant terms is selected based on the defined process inputs and outputs by the sub model selection algorithm.

\section{Results \& Discussion}

For the dynamic die melt temperature profile prediction model selection, a number of different model combinations (i.e. with different orders and number of terms) were studied. Two past output terms and one past input term from each input were used to predict the current output (i.e. $n_{a}=2$ and $n_{b}$ for each input is equal to 1$)$. Then the maximum delays $\left(n_{k}\right)$ attributed to each model input have to be determined. Melt temperature variations at each redial position followed by screw speed and barrel set temperature changes were observed from the measured signals. Based on these observations reasonable values were assumed for delays attributed to each input as: $d_{\omega_{s c}}=10 \mathrm{~s}, d_{R p}=0 \mathrm{~s}, d_{T 1}=150 \mathrm{~s}, d_{T 2}=120$ $\mathrm{s}, d_{T 3}=90 \mathrm{~s}$, and $d_{T 4}=60 \mathrm{~s}$. These delays can be adjusted as required depending on the screw geometry, material, processing conditions, etc.

To test the accuracy, the root mean squared errors (RMSE) and the normalised prediction errors (NPE) of the models were determined by equations (19) and (20) respectively.

$$
\begin{gathered}
R M S E=\sqrt{\frac{1}{N} \sum_{i=1}^{N}\left[\hat{y}_{i}(t)-y_{i}(t)\right]^{2}} \\
N P E \triangleq\left[\sum_{i=1}^{N}\left(\hat{y}_{i}(t)-y_{i}(t)\right)^{2} / \sum_{i=1}^{N} y_{i}(t)^{2}\right]^{1 / 2} \times 100 \%
\end{gathered}
$$

where $y_{i}(t)$ and $\hat{y}_{i}(t)$ are the measured and model estimated melt temperatures at time $t$ respectively, and $N$ is the number of data points. The details of some of the models studied are shown in Table II along with their RMSE and NPE on the unseen data. As shown in Table II, RMSE or NPE of the models did not improve much with increases in the order or number of terms.

$$
\begin{aligned}
\hat{T}_{m, j}(t) & =1.6128 \times \hat{T}_{m, j}(t-1)-0.0048 \times \hat{T}_{m, j}(t-1) \times R_{p, j} \\
& +0.0071 \times R_{p, j} \times T_{4}(t-60)-0.0870 \times \hat{T}_{m, j}(t-2) \\
& +0.0018 \times \omega_{s c}(t-10) \times R_{p, j}+0.0001 \times T_{3}(t-90)^{2} \\
& -0.0080 \times \hat{T}_{m, j}(t-1) \times T_{1}(t-150)-0.4998 \times T_{4}(t-60) \\
& -0.0045 \times \hat{T}_{m, j}(t-2) \times \omega_{s c}(t-10)+0.2806 \times \omega_{s c}(t-10) \\
& +0.0032 \times \hat{T}_{m, j}(t-1) \times \omega_{s c}(t-10)-0.0200 \times R_{p, j}^{2} \\
& +0.0079 \times T_{1}(t-150) \times T_{4}(t-90)-0.6378 \times R_{p, j}
\end{aligned}
$$

However, finally a $2^{\text {nd }}$ order model with 14 terms was selected for the discussion as presented in equation (21). Simulation results from this nonlinear model shows a $80.06 \%$ fit (RMSE=2.62) with the training data and a $78.24 \%$ fit (RMSE=2.86) with the unseen data.

TABLE II

RMSE AND NPE (WITH UNSEEN DATA) OF THE STUDIED NONLINEAR MODELS WITH DIFFERENT ORDERS AND NUMBER OF TERMS

\begin{tabular}{|c|c|c|c|c|}
\hline \multirow{2}{*}{\begin{tabular}{c} 
Number $\begin{array}{c}\text { of } \\
\text { terms }\end{array}$ \\
\cline { 2 - 5 }
\end{tabular}} & \multicolumn{2}{|c|}{ RMSE and NPE with model order } \\
\cline { 2 - 5 } & RMSE & NPE (\%) & RMSE & NPE (\%) \\
\hline \hline 1 & 4.499 & 1.976 & 4.499 & 1.976 \\
\hline 2 & 3.492 & 1.534 & 3.484 & 1.530 \\
\hline 3 & 3.410 & 1.498 & 3.288 & 1.444 \\
\hline 4 & 3.320 & 1.458 & 3.263 & 1.433 \\
\hline 5 & 3.309 & 1.453 & 3.195 & 1.404 \\
\hline 6 & 3.312 & 1.455 & 3.046 & 1.338 \\
\hline 7 & 3.310 & 1.454 & 3.024 & 1.328 \\
\hline 8 & 3.313 & 1.455 & 2.962 & 1.301 \\
\hline 9 & 3.313 & 1.455 & 2.956 & 1.299 \\
\hline 10 & 3.313 & 1.455 & 2.933 & 1.288 \\
\hline 11 & 3.313 & 1.455 & 2.916 & 1.281 \\
\hline 12 & 3.313 & 1.455 & 2.882 & 1.266 \\
\hline 13 & 3.313 & 1.455 & 2.864 & 1.258 \\
\hline 14 & 3.313 & 1.455 & $\mathbf{2 . 8 5 5}$ & $\mathbf{1 . 2 5 4}$ \\
\hline 15 & 3.313 & 1.455 & 2.828 & 1.242 \\
\hline
\end{tabular}

The measured and the model estimated temperature profiles over 200 data points with test condition $\mathrm{C}$ are shown in Figure 7 together with the modelling error (ME).

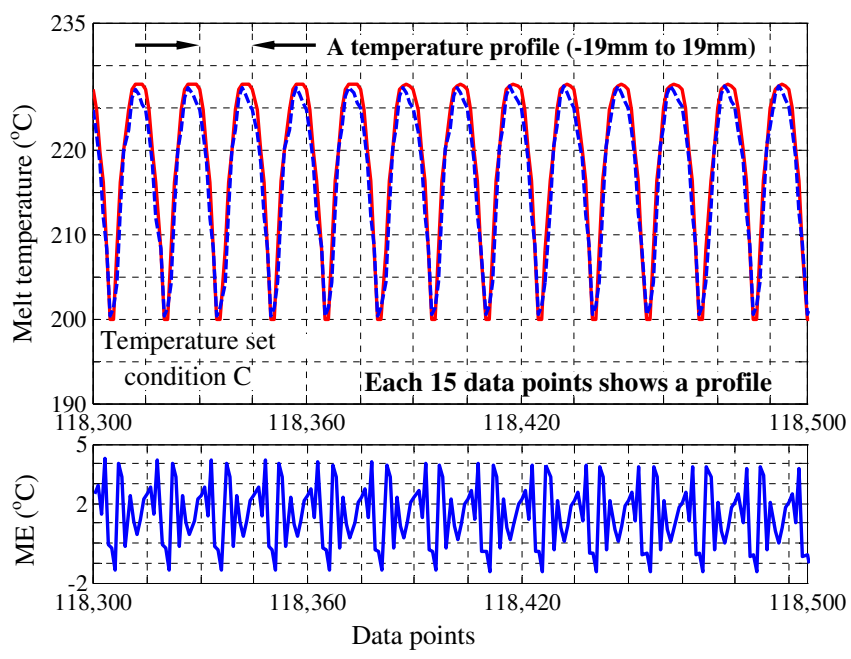

Fig. 7. Estimated and measured melt temperature profiles with modelling error for test condition $\mathrm{C}$ over 200 data points

It is evident that the model can predict the real-time die melt temperature profile with good accuracy. Then, each model term was closely examined to explore the effects of the individual process input on the die melt temperature profile. The screw speed $\left(\omega_{s c}\right)$ was identified as the most influential processing parameter on process thermal conditions particularly on the level of the output melt temperature. Of the barrel zone temperatures, $T_{4}$ seems to be the most significant zone on the die melt flow homogeneity and the temperature level under dynamic conditions while $T_{1}$ also has significant effects. As shown in equation (21), $R_{p}$ is included within several model terms which emphasises the significance of the radial position specific thermal variability of melt flow. 
According to the previous findings of Rasid and Wood [9] and Crabtree et al. [27], screw speed was the most influential process parameter on the level of the extruder output melt temperature while the metering zone temperature was the most significant barrel zone temperature. They performed experiments on industrial extruders by manually changing each process parameter to investigate their effects on the level of the extruder output melt temperature. The extruder used for this study has four temperature zones and a significant proportion of zone $3\left(T_{3}\right)$ and all of zone $4\left(T_{4}\right)$ control the temperature in the metering zone (see Figure 2) and hence the results show an agreement with the findings of Rasid and Wood as well as the Crabtree et al. However, a previous work by the authors [5] developed a static model to predict the die melt temperature profile with a gradual compression screw and found that the melting zone temperature $\left(T_{2}\right)$ was the most significant barrel zone temperature on the die melt flow homogeneity while the metering zone temperature $\left(T_{4}\right)$ showed only slight effects. These differences may be attributed to changes in processing behaviour at dynamic and static conditions and also to the effects of different screw geometry and material.

\section{CONCLUSIONS AND FUTURE WORK}

\section{A. Conclusions}

A thermocouple mesh technique was used to monitor the dynamic melt temperature profile of an industrial polymer extrusion plant. A novel dynamic polynomial model to predict the die melt temperature profile from readily measurable process parameters has been presented. As shown from the model, screw speed is the most significant process parameter affecting the level of melt temperature and temperature homogeneity across the melt flow while the metering zone temperature is the most significant among the barrel zone temperatures. These predictions of the proposed model are in agreement with previously reported experimental findings.

\section{B. Future Work}

In future work, the model will be used to establish the optimum process settings to minimise melt flow nonhomogeneity under dynamic conditions. Consideration of the effects of material properties and machine geometrical parameters should help to improve the model performance further. Also, the dynamic model proposed seems to be representing actual process conditions with good accuracy over a wide operating window and hence this will help to demonstrate a potential method for determining melt flow homogeneity in-process (i.e. an industrial soft sensor) and to build-up a control strategy to obtain the required melt flow homogeneity in extrusion by manipulating the process settings while maintaining the required mass throughput and average die melt temperature.

\section{ACKNOWLEDGMENTS}

This work was funded through multi-disciplinary research programmes (Grant Nos: EP/G059330/1 and EP/F021070/1) by the Engineering and Physical Sciences Research Council, UK.

\section{REFERENCES}

[1] S. Dormeier and K. Panreck, "Dynamic behaviour of the temperature during extrusion," Kunststoffe, vol. 80, no. 11, pp. 32-33, 1990.

[2] C. Abeykoon, Polymer Extrusion: A Study on Thermal Monitoring Techniques and Melting Issues. Lap Lambert Publishing:Verlag, 2012.

[3] C. Abeykoon, P. J. Martin, A. L. Kelly, and E. C. Brown, "An evaluation of melt temperature sensors for polymer extrusion," Sens. Actuators, A, vol. 182, pp. 16-27, 2012.

[4] C. Abeykoon, "Modelling and control of melt tempearture in polymer extrusion," Ph.D. dissertation, Queen's University Belfast, UK, 2011.

[5] C. Abeykoon, K. Li, M. McAfee, P. J. Martin, Q. Niu, A. L. Kelly, and J. Deng, "A new model based approach for the prediction and optimisation of thermal homogeneity in single screw extrusion," Control Eng. Pract., vol. 19, no. 8, pp. 862-874, 2011.

[6] C. Abeykoon, M. McAfee, K. Li, A. L. Kelly, and E. C. Brown, "Monitoring the effect of operating conditions on melt temperature homogeneity in single-screw extrusion," SPE ANTEC Tech. Papers, vol. 1, pp. 1799-1806, 2010.

[7] A. L. Kelly, E. C. Brown, and P. D. Coates, "The effect of screw geometry on melt temperature profile in single screw extrusion," Polym. Eng. Sci., vol. 46, no. 12, pp. 1706-1714, 2006.

[8] E. C. Brown, A. L. Kelly, and P. D. Coates, "Melt temperature field measurement in single screw extrusion using thermocouple meshes," Rev. Sci. Instrum., vol. 75, no. 11, pp. 4742-4748, 2004.

[9] R. Rasid and A. K. Wood, "Effect of process variables on melt velocity profiles in extrusion process using single screw plastics extruder," Plast. Rubber Compos., vol. 32, no. 5, pp. 193-198, 2003.

[10] X. Shen, R. Malloy, and J. Pacini, "An experimental evaluation of melt tempearture sensors for thermopalstic extrusion," SPE ANTEC Tech. Papers, pp. 918-926, 1992.

[11] C. Rauwendaal, Polymer extrusion. Hanser-Munich, 2001.

[12] A. J. Bur, M. G. Vangel, and S. C. Roth, "Fluorescence based temperature measurements and applications to real-time polymer processing," Polym. Eng. Sci., vol. 41, no. 8, pp. 1380-1389, 2001.

[13] W. Fontaine, Ph.D. dissertation, Ohio State University, Ohio, 1975.

[14] D. H. Reber, R. E. Lynn, and E. J. Freeh, "A mathematical model for predicting dynamic behavior of a plasticating extruder," Polym. Eng. Sci., vol. 13, no. 5, pp. 346-356, 1973.

[15] Z. Tadmor, S. Lipshitz, and R. Lavie, "Dynamic model of a plasticating extruder," Polym. Eng. Sci., vol. 14, no. 2, pp. 112-119, 1974.

[16] A. Kochhar and J. Parnaby, "Dynamical modelling and control of plastics extrusion processes," Automatica, vol. 13, no. 2, pp. 177-183, 1977.

[17] D. D. Germsuka, P. A. Taylor, and J. D. Wright, "Adaptive and multivariable control of a single screw extrusion system," Can. J. Chem. Eng., vol. 62, no. 6, pp. 790-801, 1984.

[18] D. Chan, R. W. Nelson, and L. J. Lee, "Dynamic behavior of a single screw plasticating extruder part II: dynamic modeling," Polym. Eng. Sci., vol. 26, no. 2, pp. 152-161, 1986.

[19] Y. J. Lin and G. Lee, "System identification for state feedback integral observer control of polymer plastic extrusion," Polym. Plast. Technol. Eng., vol. 36, no. 5, pp. 749-775, 1997.

[20] L. P. Tan, A. Lotfi, E. Lai, and J. B. Hull, "Soft computing applications in dynamic model identification of polymer extrusion process," Appl. Soft Comput., vol. 4, no. 4, pp. 345-355, 2004.

[21] J. D. Bomberger and D. E. Seborg, "Determination of model order for narx models directly from input-output data," J. Process Control, vol. 8, no. 5-6, pp. 459-468, 1998.

[22] C. Abeykoon, K. Li, M. McAfee, P. J. Martin, J. Deng, and A. L. Kelly, "Modelling the effects of operating conditions on on die melt temperature homogeneity in single screw extrusion," UKACC Int. Conf. on Control, Coventry, United Kingdom, pp. 42-47, 2010.

[23] K. Li, J. X. Peng, and G. Irwin, "A fast nonlinear model identification method," IEEE Trans. Autom. Control, vol. 50, pp. 1211-1216, 2005.

[24] K. Li, J. X. Peng, and E. W. Bai, "A two-stage algorithm for identification of nonlinear dynamic systems," Automatica, vol. 42, no. 7, pp. 1189-1197, 2006.

[25] S. Chen, S. Billings, and W. Luo, "Orthogonal least squares methods and their application to non-linear system identification," Int. J. Control, vol. 50, pp. 1873-1896, 1989.

[26] O. Nelles, Nonlinear System Identification. Springer-Verlag, 2001.

[27] S. L. Crabtree, M. A. Spalding, and C. L. Pavlicek, "Single-screw extruder zone temperature selection for optimized performance," $S P E$ ANTEC Tech. Papers, vol. 3, pp. 1406-1411, 2008. 